\title{
Evapotranspiración del cultivo de granado por balance de energía
}

\section{Evapotranspiration of pomegranate crop by energy balance}

\author{
SIFUENTES-MORÍN, Norma Guadalupe†*, FRÍAS-RAMÍREZ, José Ernesto, SERVÍN-PRIETO, Alan \\ Joel y MONTEMAYOR-TREJO, José Alfredo
}

\author{
Instituto Tecnológico de Torreón. Carretera Torreón-San Pedro de las Colonias Km 7.5, Ejido Ana, CP 27170. Torreón, \\ Coah, México. \\ ID 1 ${ }^{\mathrm{er}}$ Autor: Norma Guadalupe, Sifuentes-Morín / ORC ID: 0000-0003-4724-5294, CVU CONACYT ID: 713430 \\ ID 1 ${ }^{\text {er }}$ Coautor: José Ernesto, Frías-Ramírez / ORC ID: 0000-0002-6631-6599, CVU CONACYT ID: 67627 \\ ID $2^{\text {do }}$ Coautor: Alan Joel, Servín-Prieto / ORC ID: 0000-0002-5534-7875, CVU CONACYT ID: 255753 \\ ID $3^{\text {er }}$ Coautor: José Alfredo, Montemayor-Trejo / ORC ID: 0000-0001-8222-286X, CVU CONACYT ID: 201396
}

DOI: $10.35429 /$ JSL.2020.22.7.1.6

Recibido 10 de Enero, 2020; Aceptado 30 de Junio, 2020

\section{Resumen}

La evapotranspiración (ET), es un elemento clave en el cálculo del balance de energía superficial, en la detección de estrés hídrico y también en cálculo del rendimiento agrícola. Sin embargo, su medición directa o, su estimación es muchas veces complicada dada la diversidad y complejidad de los factores que actúan en el proceso (morfológicos, fisiológicos, edafológicos). El método SEBAL (Algoritmo de balance de energía superficial de la tierra), estima la ET a través de imágenes de satélite, utilizando los principios del balance de energía superficial, con el que se han obtenido excelentes resultados como lo reportan estudios realizados por diferentes autores; minimizando de esta forma los costos y el tiempo de estimación de la ET en zonas extensas de vegetación. El objetivo principal de esta investigación fue estimar la evapotranspiración potencial de cultivo de granado en una huerta comercial ubicada en el municipio de Gómez Palacio, Durango, México. El método SEBAL fue aplicado con imágenes satelitales Landsat 8 , durante el ciclo de cultivo. Los resultados se validaron con datos de ET estimados con el método FAO 56 obteniéndose un índice de concordancia de Willmott de 0.96, lo que significa buena precisión de estimación.

Evapotranspiración, SEBAL, Granado

\begin{abstract}
Evapotranspiration is a key element in calculating the surface energy balance, wáter balance and crop water stress and crop yield determination. However, it's direct measurement or estimation is frequently complicated, since the diversity and complexity of the factors acting in this physical process. (morphological, physiological and soil factors). SEBAL (Surface Energy Balance Algorithm for Land) estimates ET based on satellite images, using the principles of surface energy balance producing excellent results as reported in several studies of different scientist authors; minimizing the cost and time for the ET determination for large vegetation zones. The objective of this research work was to estimate the potential evapotranspiration for the pomegranate crop in a commercial farm, located in Gomez Palacio, Durango, Mexico, by SEBAL using Landsat 8 satellite images during the crop cycle 2016. The results were validated with estimates of ET by the FAO 56 method, obtaining a Willmott concordance index of 0.96 , which means good estimation precision.
\end{abstract}

Evapotranspiration, SEBAL, Pomegranate

Citación: SIFUENTES-MORÍN, Norma Guadalupe, FRÍAS-RAMÍREZ, José Ernesto, SERVÍN-PRIETO, Alan Joel y MONTEMAYOR-TREJO, José Alfredo. Evapotranspiración del cultivo de granado por balance de energía. Revista de Simulación y Laboratorio 2020. 7-22:1-6. 


\section{Introducción}

Uno de los elementos más transcendentales que influyen en la producción y rendimiento del cultivo es la ET, está un elemento clave en el cálculo del balance de energía superficial, en el balance hídrico, en la detección de estrés hídrico y también en cálculo de los rendimientos agrícolas (Cleves et al., 2016).

Sin embargo, su medición directa o, su estimación es muchas veces complicada dada la diversidad y complejidad de los factores que actúan en el proceso (meteorológicos, fisiológicos, edafológicos) (Sánchez, 2002).

Uno de los métodos más utilizados actualmente en México para calcular la ET es con el método FAO 56, basado en la ecuación de Penman Monteith, con la que se obtienen excelentes resultados gracias a las múltiples variables y complejidad que presentan estos modelos. (FAO, 2012).

Estos modelos requieren de una serie de datos de estaciones meteorológicas distribuidas estratégicamente en la zona de estudio lo cual es costoso y poco viable para zonas de extensos cultivos. (FAO, 2006). Por lo anterior, surge la necesidad de buscar herramientas que permitan facilitar las estimaciones y monitoreo de parámetros biofísicos, que inciden de forma directa con la producción y calidad de los cultivos. Una herramienta que facilita y mejora las estimaciones de la ET es la teledetección, la cual ha presenta avances y resultados significativos en el cálculo de fenómenos que afectan la producción agrícola en los últimos años. (Zang, 2016).

La teledetección es una fuente de información que puede emplearse para obtener estimaciones de ET a distintas escalas, tanto espaciales como temporales, (Gómez, 2005), que se fundamenta en procesos físicos que pueden ser observados a través de la teledetección. Un ejemplo de esto es el algoritmo SEBAL (Balance de energía de superficie para tierra) desarrollado por Bastiaanssen en 1995.
Este método estima la ET a través de imágenes de satélite, utilizando los principios del balance de energía superficial, con el que se han obtenido excelentes resultados en diversos estudios realizados por diferentes autores como; (Allen R. P., 2006), (Castañeda, 2013), (Wang J, 2009), (Trezza R., 2008) minimiza de esta forma los costos y el tiempo de estimación de la ET en zonas extensas de vegetación.

Los objetivos del presente estudio fueron estimar la ET utilizando imágenes, así como, validar las estimaciones con valores de ET obtenidos con datos de estación climatológicas locales.

\section{Metodología}

\section{Localización y cultivo}

El estudio se realizó en el rancho "El Triángulo" en Gómez Palacio Durango, México, con coordenadas $25^{\circ} 37^{\prime} 41^{\prime \prime}$ Norte $103^{\circ} 26^{\prime} 54^{\prime \prime}$ Oeste, y con una altitud de $1123 \mathrm{msnm}$.

Para el estudio fue considerada una superficie de 20 hectáreas, con árboles de granado variedad Wonderful, de dos años de edad. Los frutales están establecidos en un sistema de siembra de tres bolillos, con separaciones de tres por cinco metros, a una densidad de plantación de 666 árboles ha-1. Los árboles son regados con un sistema riego por goteo.

\section{Estimación de la Evapotranspiración potencial (Etp) por el método FAO 56, 2006.}

La evapotranspiración potencial se estimó con datos de una estación agrometeorológica ubicada en el Centro Nacional de Investigación Interdiciplinaria relación Agua Suelo Planta del INIFAP, ubicada a km 6.5 margen derecho del Canal Sacramento en Gómez Palacio Durango. La estación climatológica usada fue modelo Davis. Los datos corresponden al periodo comprendido del 15 de febrero al 15 de noviembre del 2016. 


\section{Estimación de la ET instantánea con SEBAL}

Para la estimación de la ET mediante Balance de Energía superficial se utilizaron imágenes del satélite Landsat 8 adquirida por los sensores OLI y TIR, mismas que fueron obtenidas desde el portal del Servicio Geológico de los Estados Unidos (USGS). http://earthexplorer.usgs.gov/. Además, se utilizó un modelo digital de elevación a $30 \mathrm{~m}$ obtenido a partir del portal del Instituto Nacional de Estadística y Geografía (INEGI). El preprocesamiento de las imágenes se llevó a cabo con el software ArcGis 10.3, el cual permite utilizar una serie de herramientas diseñadas especialmente para procesar imágenes de satélite.

Primero se convirtieron los valores de niveles digitales (ND) de cada píxel a parámetros físicos para obtener la radiancia espectral y la reflectancia, dichos parámetros representan las bases físicas para el procesamiento de SEBAL a través de la herramienta de calculadora ráster.

\begin{tabular}{|c|r|}
\hline \multicolumn{1}{|c|}{ Fecha } & Path/Row \\
\hline $18 / 02 / 2016$ & $30 / 42$ \\
\hline $05 / 03 / 2016$ & $30 / 42$ \\
\hline $21 / 03 / 2016$ & $30 / 42$ \\
\hline $09 / 06 / 2016$ & $30 / 42$ \\
\hline $25 / 06 / 2016$ & $30 / 42$ \\
\hline $11 / 07 / 2016$ & $30 / 42$ \\
\hline $12 / 08 / 2016$ & $30 / 42$ \\
\hline $13 / 09 / 2016$ & $30 / 42$ \\
\hline $15 / 10 / 2016$ & $30 / 42$ \\
\hline $31 / 10 / 2016$ & $30 / 42$ \\
\hline $16 / 11 / 2016$ & $30 / 42$ \\
\hline
\end{tabular}

Tabla 1 Imágenes Landsat utilizadas en el estudio Fuente: Elaboración propia

Se realizo todo el procedimiento del Modelo SEBAL (Bastiaanssen, W, 1995). hasta la obtención de la ET instantánea como se muestra en la figura 1.

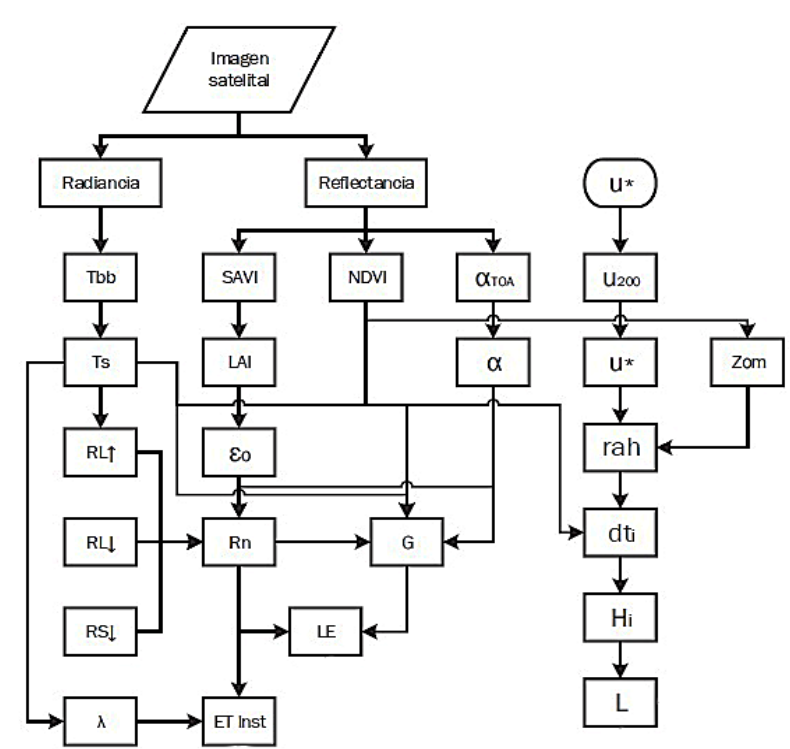

Figura 1 Procesamiento de generación de parámetros de SEBAL

Fuente: Delgado, 2017

\section{Análisis estadístico}

La estimación de ET instantánea se compararon contra los valores obtenidos por el método FAO 56, utilizado regresión lineal y el índice de concordancia Will Willmott (1982). (Cai, 2007). Para determinar la precisión de las estimaciones, considerando índices de uno como concordancia perfecta, mientras que un valor de cero refleja una no concordancia.

Para evaluar la función lineal se utilizó el coeficiente de determinación $\left(\mathrm{R}^{2}\right)$, la pendiente $\mathrm{b}$ de la regresión lineal entre valores de FAO 56 y estimados con SEBAL y, además, los siguientes estadísticos: la desviación media del error (MBE), la raíz cuadrada del error cuadrático medio (RMSE), el error relativo (RE), el índice de concordancia de Willmott ( Willmott, 1981)

\section{Resultados}

Los valores de ET estimados mediante SEBAL y los estimados por el método de FAO 56, se muestran en la Tabla 2. Las evapotranspiraciones estimadas mediante FAO 56 corresponden a las 6:00 pasado meridiano (P.M.), dado que es la hora más cercana al paso del satélite. 


\begin{tabular}{|c|r|r|r|}
\hline Fecha & Dia juliano & \multicolumn{3}{|c|}{$\begin{array}{c}\text { ETPenman } \\
\mathbf{m m} / \mathbf{h r}\end{array}$} & $\begin{array}{c}\text { ETmax_L } \\
\mathbf{m m} / \mathbf{h r}\end{array}$ \\
\hline $05 / 03 / 2016$ & 65 & 0.36 & 0.36 \\
\hline $21 / 03 / 2016$ & 81 & 0.38 & 0.36 \\
\hline $09 / 06 / 2016$ & 161 & 0.53 & 0.58 \\
\hline $25 / 06 / 2016$ & 177 & 0.53 & 0.53 \\
\hline $11 / 07 / 2016$ & 193 & 0.58 & 0.57 \\
\hline $12 / 08 / 2016$ & 225 & 0.43 & 0.46 \\
\hline $13 / 09 / 2016$ & 257 & 0.33 & 0.31 \\
\hline $15 / 10 / 2016$ & 289 & 0.3 & 0.18 \\
\hline $31 / 10 / 2016$ & 305 & 0.25 & 0.17 \\
\hline $16 / 11 / 2016$ & 321 & 0.25 & 0.23 \\
\hline
\end{tabular}

Table 2 Estimaciones de ET con los métodos FAO 56 y SEBAL

Fuente: Elaboración propia

Dadas estas variaciones en las estimaciones de ET, se encontró que para el 27\% de las imágenes procesadas con SEBAL (día juliano 161, 177 y 225), (Figura 2), se reporta un valor mayor del estimado con Penman con $0.0503,0.002$, y $0.0303 \mathrm{~mm}$ respectivamente, mientras que para las siete imágenes restantes (día juliano 65, 81, 193, 257, 289, 305 y 321) el valor de ET se subestima, con 0.0026, 0.0181, $0.0091, \quad 0.0214, \quad 0.1194, \quad 0.0847 \mathrm{y} \quad 0.0192$ respectivamente Las diferencias más significativas se presentan en octubre, en los días julianos 289 y 305.

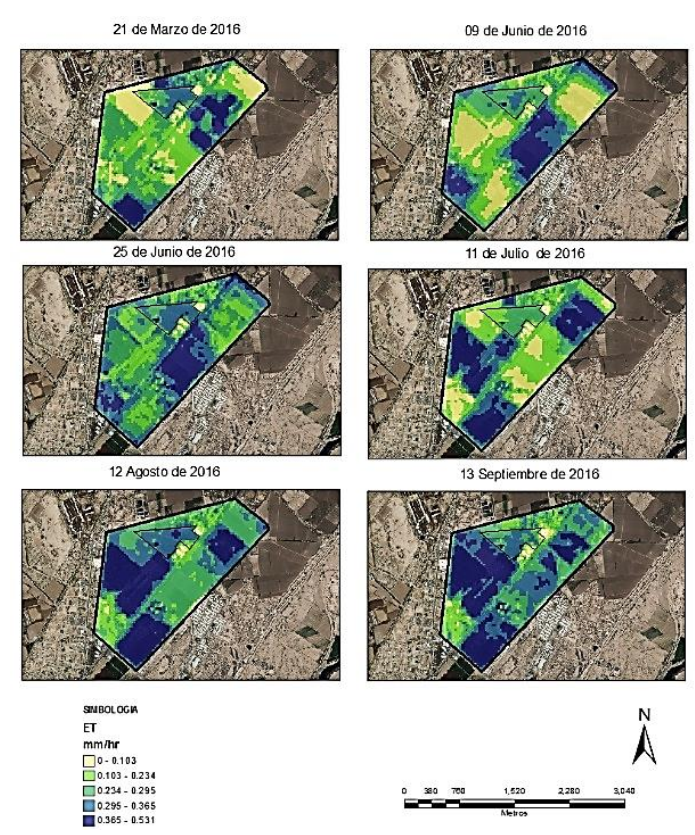

Figura 2 Variación de evapotranspiración por el método SEBAL en el área de estudio Fuente: Elaboración propia

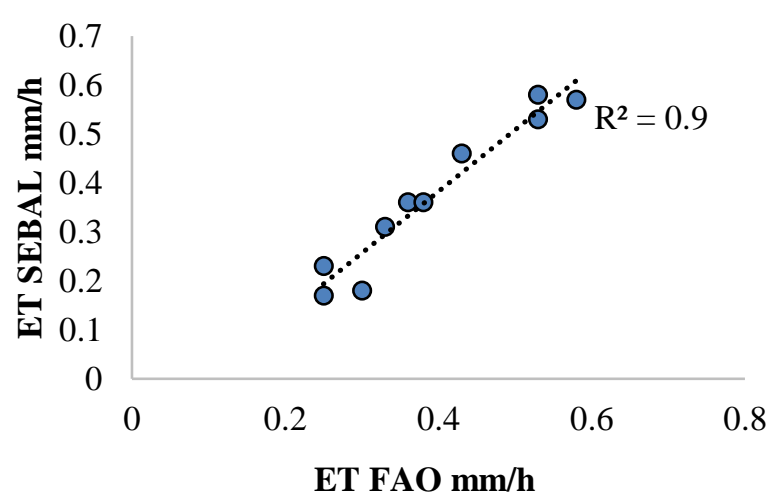

Gráfico 1 Modelo de regresión lineal de la ET estimada entre los métodos FAO 56 versus SEBAL

Fuente: Elaboración propia

El gráfico 1 muestra la relación que existe entre la ET estimada con el método FAO 56 versus SEBAL. Se encontró una alta correlación entre ambos métodos, ya que para las 11 imágenes se ajustan a modelo lineal con un coeficiente de determinación $\mathrm{R}^{2}=0.94$.

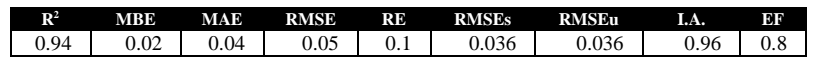

Tabla 3 Comparación de métodos con el índice de concordancia de Willmot

Fuente: Elaboración propia

En la tabla 3 se muestran los valores obtenidos para el cálculo del índice de concordancia (I.A.); su valor es de 0.96, el cual nos indica una buena concordancia de la Et estimada entre ambos métodos, puesto que un valor de 1 que es una concordancia perfecta, mientras que un valor de 0 refleja una concordancia pobre y la eficiencia del método SEBAL fue de 0.80 con respecto al FAO 56, el cual es considerado como bueno estadísticamente. La eficiencia del método de SEBAL estadísticamente fue de 0.80 con respecto al FAO 56, considerado como bueno.

\section{Conclusiones}

De acuerdo con los resultados en el presente estudio se puede concluir lo siguiente:

Se confirma que el método de SEBAL es una herramienta muy útil para la estimación de Et con imágenes satelitales.

El método de SEBAL permite hacer análisis puntual, espacial y temporal de la ET, representando ventajas en costos de equipos y en la limitada disponibilidad de datos de clima. 
Los resultados sugieren que la metodología propuesta puede implementarse con facilidad y con muy buenos resultados, ya que obtuvo un alto índice de concordancia en comparación a los calculados del método FAO.

\section{Referencias}

Allen, R. P. (2006). Evapotranspiración del Cultivo: Guías para la Determinación de Agua de los Cultivos. Estudio FAO Riego y Drenaje. FAO Organización de las Naciones Unidas para la Agricultura y la Alimentación.

Allen, R., Perreira, R., \& Rae, D. (1998). Crop evapotranspiration: Guidelines for computing crop water requirements. In FAO Irrigation and Drainage Paper 56, pp.135-56.

Allen, R., Tasumi, M., \& Bastiaanssen, R. (2002). SEBAL, Surface Energy Balance Algorithms for Land. Advance Training and Users. En Manual. Idaho: a NASA EOSDIS/Synergy grant from the Raytheon Company University of Idaho.

Bastiaanssen, W. (1995). Regionalization of surface flux desities and moisture indicators in composite terrain: a remote sensing approach under clear skies in Mediterranean climates. Land bouw universiteit te Wageningen, 109, p. 273 .

Cai, J., Liu, Y., Lei , T., \& Pereira, L. (2007). Estimating reference evapotranspiration with the FAO Penman-Monteith equation using daily weather forecast messages. Agricultural and Forest Meteorology, 145(1-2):22-35.

Castañeda, C. (2013). Estimación de la evapotranspiración mediante un balance de energía utilizando sensores remotos. . Tesis para optar el título de Maestro en Ciencias. Montexillo, México: Institución de enseñanza e investigación en Ciencias Agrícolas. .

Chuvieco, E. (2006). Teledetección Ambiental. La Observación de la Tierra desde el Espacio. 2nd ed. Ariel, S.A.
Cleves L., J., Toro C., J., \& Martínez B., L. (2016). Los balances hídricos agrícolas en modelos de simulación agroclimáticos. Una revisión analítica. Revista Colombiana De Ciencias Hortícolas, 10(1), 149-163. https://doi.org/10.17584/rcch.2016v10i1.4460

FAO, 1989. Irrigation water management, Training manuals. Disponible en: http://www.fao.org/docrep/T7202E/T7202E00. htm.

FAO, 2006. Evapotranspiracíon del cultivo, Guías para la determinación de los requerimientos de agua de los cultivos. 9253042192nd ed. Rome, Italia: ESTUDIO FAO RIEGO Y DRENAJE. Disponible en: ftp://ftp.fao.org/agl/aglw/docs/idp56s.pdf.

FAO, 2012. Organización de las Naciones Unidad para la Alimentación y la Agricultura. [En linea] (1) Disponible en: http://www.fao.org/statistics/es/

Gómez, M. O. (2005). . Retrieval of evapotranspiration over the Alpilles/ReSeDA experimental site using airborne Polder sensor and a thermal Camera. Remote Sensing of Environment, pp.399408. .

González, M., Treviño, E., Aguirre, O., Jimenez, J., Cantú, I., \& Foroughbakhch , R. (2004). Rodalización mediante sistemas de información. Investigaciones Geográficas, Boletín 53 40-57.

Henríquez Dole, L., \& Miner Vega, A. (2015). Revisión y validación de la evapotranspiración potencial (ETP) media anual y media mensual en Honduras obtenida por métodos ambientales de percepción remota. Revista Ciencia Y Tecnología, (14), 77-98. https://doi.org/10.5377/rct.v0i14.1797

Delgado Ramírez, G., Estrada Ávalos, J., Rivera González, M., Miguel Valle, E., Servín Prieto, A., y, Ramos Cruz, C. 2017. Procedimiento metodológico para estimar necesidades hídricas en cultivos mediante imágenes de satélite y modelo sebal. Folleto Técnico No. 44. CENIDRASPA. Gómez Palacio, Durango. ISBN: 978607-37-0902-6. 47 p.

Molina. (2009). Estandarización de las ecuaciones para estimar la evapotranspiración del cultivo de referencia. Ingeniería, Investigación y Tecnología., 10(2): 125-135. 
Sanchez, M. M. (2002). Modelos y Aproximaciones para la Estimación de la Evapotranspiración con Información Satelital. Revista de Geografía Norte Grande,, pp.107-20.

Tasumi, M. (2003). Progress in operational estimation of regional evapotranspiration using satellite imagery. Dissertation Abstracts International, 64(2), p.357.

Trezza, R. (2008). Estimación de evapotranspiración de referencia a nivel mensual en venezuela. ¿cuál método utilizar? . Bioagro, 2(20), pp.89-95. .

USGS. (2016). Obtenido de

https://www.usgs.gov/

Wang J, J. e. (2009). Sensivility analysis of the surface energy balance algorithm for land (SEBAL). . American Society of Agricultural and Biological Engineers ISSN, 52(3), pp.80111 .

Willmott, C. J. (1981). On the validation of models. Physical Geography, 2(2), 184194. doi:10.1080/02723646.1981.10642213

Zhang, K., Kimball, J. S., \& Running, S. W. (2016). A review of remote sensing based actual evapotranspiration estimation. Wiley 